\title{
Closing the Brief Case: Bacteremia and Vertebral Osteomyelitis Due to Staphylococcus schleiferi
}

\author{
Melanie L. Yarbrough, ${ }^{a}$ Yasir Hamad,b (D) Carey-Ann D. Burnham, ${ }^{\text {age A. George }}{ }^{\text {b }}$ \\ Department of Pathology \& Immunology, Division of Laboratory and Genomic Medicine, Washington \\ University School of Medicine, St. Louis, Missouri, USAa; Department of Medicine, Division of Infectious \\ Diseases, Washington University School of Medicine, St. Louis, Missouri, USA ${ }^{b}$
}

\section{ANSWERS TO SELF-ASSESSMENT QUESTIONS}

1. What is detected by a tube coagulase test using rabbit plasma?
A. Free coagulase
B. Bound coagulase
C. Protein A
D. Hemolysin

Answer: A. The tube coagulase test detects the presence of free coagulase that is secreted by S. aureus and other coagulase-positive Staphylococcus species. The slide coagulase test detects the presence of bound coagulase (also known as clumping factor), which reacts with fibrinogen to form a clump (agglutination). Protein $A$ is a constituent of the $S$. aureus cell wall that can be detected by latex agglutination testing methods.

2. A Gram-positive coccus in clusters tests positive for catalase and PYR but negative for urease and ornithine decarboxylase. What is the most likely identity of this organism?
A. S. aureus
B. S. schleiferi subsp. schleiferi
C. S. schleiferi subsp. coagulans
D. S. lugdunensis.

Answer: B. S. schleiferi subsp. schleiferi is PYR positive and ornithine decarboxylase and urease negative. S. schleiferi subsp. schleiferi is distinct from S. schleiferi subsp. coagulans, which is tube coagulase and urease positive. S. aureus is PYR negative and S. lugdunensis is ornithine decarboxylase positive.

3. Which of the following methods is a rapid test that could be used to predict methicillin resistance in $S$. schleiferi?
A. PBP2a testing by immunochromatographic assay
B. Cefinase test
C. Cefoxitin disk diffusion
D. Oxacillin disk diffusion

Answer: A. An immunochromatographic method for detection of the PBP2a protein, which is encoded by the mecA gene, is a rapid method to predict methicillin resistance in $S$. aureus. The test can be completed in less than half an hour. This method was recently found to have good diagnostic performance with S. schleiferi isolates. While the disk diffusion method is used to predict methicillin resistance in Staphylococcus species, this method is not considered rapid, as it requires a day for bacterial growth prior to the interpretation of assay results.

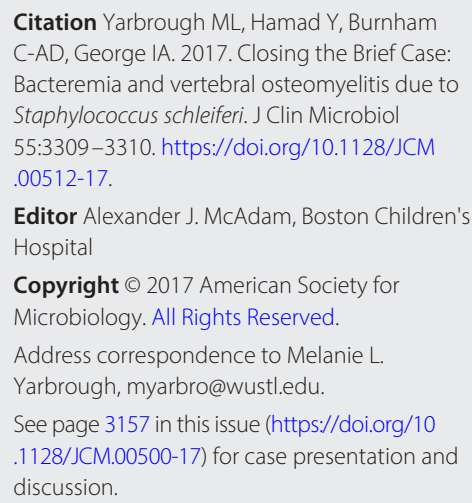


Current guidelines use cefoxitin breakpoints to predict methicillin resistance, but there is evidence to suggest that oxacillin may be the best surrogate for $S$. schleiferi. The cefinase test is a rapid method used to detect the production of $\beta$-lactamase-producing bacteria and is sometimes used to test for BlaZ activity in staphylococci but does not detect PBP modifications associated with methicillin resistance.

\section{TAKE-HOME POINTS}

- S. schleiferi is a component of the normal skin microbiota of dogs but can cause zoonotic infections.

- S. schleiferi is an uncommon but important cause of human infections that is associated with endocarditis, device infections, bacteremia, osteomyelitis, and skin and soft tissue infections.

- S. schleiferi may be mistaken for S. aureus because of characteristics such as beta-hemolysis on blood agar and a positive coagulase test. However, S. schleiferi is PYR positive, while S. aureus is PYR negative.

- Accurate identification of S. schleiferi is clinically relevant, as mecA is relatively common in this species, but methods for detection of methicillin resistance differ between staphylococcal species. 